\title{
Croissance du Sapin de Grèce : variabilité inter-provenances dans trois stations méditerranéennes françaises
}

\author{
B. FADY \\ avec la collaboration technique de M. D. VAUTHIER * \\ * INRA, Domaine expérimental du Ruscas (Var) \\ Laboratoire de Botanique et Ecologie Méditerranéenne, Université d'Aix-Marseille III, \\ Centre Saint-Jérôme, 13397 Marseille Cedex 13
}

\begin{abstract}
Summary
Height growth of the Greek Fir : variability between provenances in three experimental sites in Mediterranean France
\end{abstract}

Height growth is a very sensitive parameter by which to characterize genetic variability of the Greek Fir (Abies cephalonica Loud.). In this study, where ecovalence, relative performance and analysis of variance were used as mathematical and statistical tools, the species growth stability in three comparative experimental sites in Mediterranean France showed significant variations between populations of different origins. Bud opening rates were also shown to be significantly different between provenances. However, height growth dynamics remained comparable year after year for the great majority of provenances. Annual increment rates and inter-stationnal ecovalence were proven to be the most important characteristics of groups of provenances.

Provenances from the Parnassos and the Taygetos mountains were often slow growers and sometimes had highly unstable responses to environmental changes. Moreover, within each region, provenances' height growth patterns and drought resistance capacities were heterogenous.

On the contrary, provenances from the Mainalon and Pindos mountains displayed similar capacitics within each region. Pindos provenances had a strong height growth and a good growth stability under varying environmental conditions. Mainalon provenances, along with a very strong height growth, had remarkable drought resistance capacities as well as a very stable growth pattern. Therefore, they are highly desirable provenances for reforestation in the upper Quercus ilex and Quercus pubescens vegetation zones.

Key words: Abies cephalonica, genetic variability, provenances, height growth, growth stability, ecovalence, relative performance.

\section{Résumé}

La croissance en hauteur est un critère de choix pour mettre en évidence la variabilité génétique du Sapin de Grèce (Abies cephalonica Loud.). L'étude de sa stabilité d'expression dans trois plantations comparatives situées en zone méditerranéenne, à l'aide d'outils mathématiques tels que l'écovalence, la performance relative et l'analyse de variance, montre de très nettes variations en fonction du lieu d'origine des provenances constituant le dispositif expérimental. De même, l'étude des modalités de débourren'ent montre des différences significatives entre provenances. 
Les provenances des massifs du Parnasse et du Taygète sont souvent peu vigoureuses et parfois fortement instables. A l'inverse, les provenances du massif du Mainalon, remarquables pour leur résistance à la sécheresse, et celles du massif du Pinde sont très performantes même lorsque les conditions pédoclimatiques fluctuent.

Mots clés: Abies cephalonica, variabilité génétique, provenances, croissance en hauteur, stabilité de croissance, écovalence, performance relative.

\section{Introduction}

Le Sapin de Grèce recouvre deux formes botaniques, Abies cephalonica Loud. et Abies borisii-regis Mattf., ce dernier étant généralement considéré comme un hybride entre Abies cephalonica et Abies alba Mill. (PAnetsos, 1975). On le rencontre depuis les massifs montagneux du nord de la Grèce continentale où prédominent les formes du type $A$. borisii-regis jusqu'au sud du Péloponèse où $A$. cephalonica est seul représenté (Barbero \& Quezel, 1976). Les forêts de Sapin de Grèce ont une fourchette altitudinale de répartition comprise entre $600-700 \mathrm{~m}$ et $2200-2300 \mathrm{~m}$ (étages méso-, supra-, montagnard- et oro-méditerranéens), avec un optimum écologique entre 1000 et $2000 \mathrm{~m}$ d'altitude. Elles colonisent des sols sur roches mères aussi variées que les calcaires et les dolomies ( $A$. cephalonica surtout) et les flyshs ( $A$. borisii-regis surtout) et se développent en ambiance bioclimatique humide allant des variantes thermiques extrêmement froide à fraîche (le paramètre $m$ du climagramme d'Emberger varie de $-10 \mathrm{C}$ à $+3 \mathrm{C}$ ), ce qui est considérable pour un Sapin méditerranéen. Le Sapin du roi Boris a une répartition plus restreinte et plus typique de celle des sapins méditerranéens puisqu'il n'est présent qu'aux étages supra- et montagnard-méditerranéens et seulement dans les variantes thermiques froide et extrêmement froide du bioclimat humide.

Sa remarquable plasticité, sa résistance aux rigueurs du climat méditerranéen ainsi que sa bonne régénération naturelle (PAULY, 1962) permettent de considérer le Sapin de Grèce comme un arbre de choix en vue de reboisements dans les forêts méditerranéennes dégradées du sud de la France.

Cependant, les massifs forestiers de l'aire d'origine étant souvent situés dans des conditions écologiques variées et isolés les uns des autres (fig. 1), la variabilité génétique de l'espèce est a priori importante. Il est donc nécessaire, avant toute chose, de bien en mesurer l'ampleur. Un des moyens d'y parvenir est de comparer entr'elles diverses provenances de l'aire d'origine sur un même terrain d'étude.

\section{Matériel et méthodes}

\subsection{Dispositif expérimental et mesures}

Le dispositif expérimental «Sapins Méditerranéens" a été créé dans les années 1960 à l'initiative des chercheurs du département des Recherches forestières de l'INRA et grâce à leur collaboration avec des forestiers de divers pays méditerranéens, principa- 


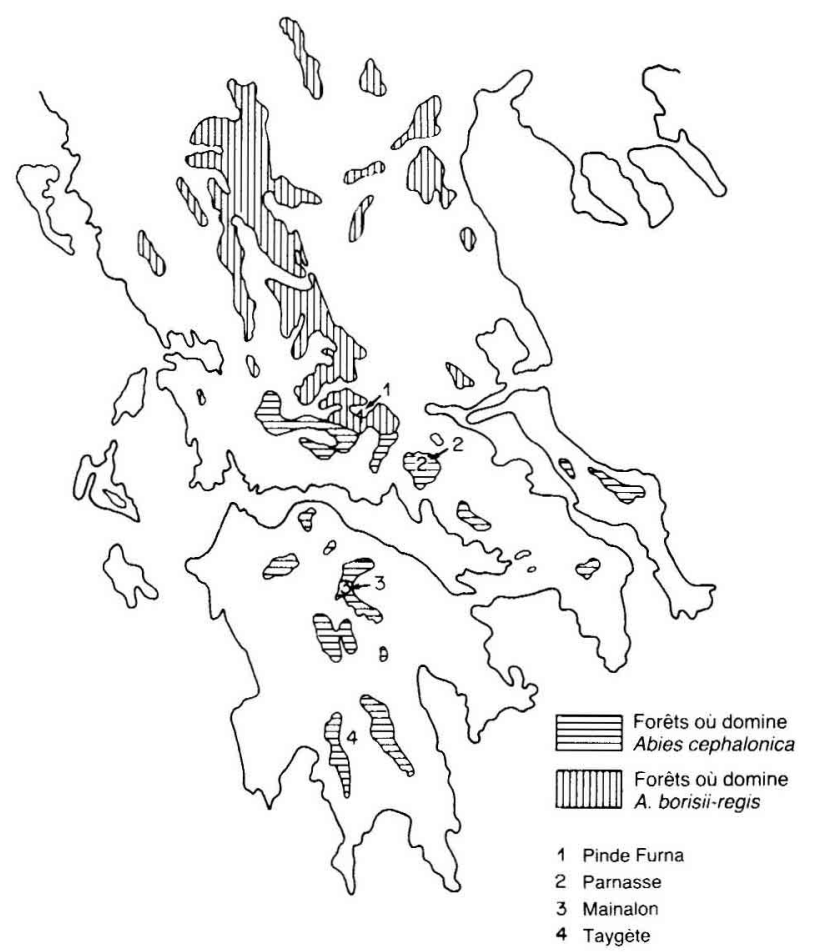

FIG. 1

Aire de répartition du Sapin de Grèce (d'après Quezel \& Barbero, 1985).

Natural distribution of the Greek Fir (from QUEZEL \& BARBERO, 1985).

lement la Grèce, pour l'échantillonnage dans l'aire d'origine et la récolte des graines. L'élevage des plants, leur mise en place sur le terrain et le suivi des mesures ont été réalisés par l'équipe INRA du Domaine Expérimental du Ruscas (Var).

Les provenances de Sapin de Grèce sont au nombre de 12 (tabl. 1), dont une ne faisant pas partie de laire naturelle, réparties sur 3 plantations comparatives: Le Treps, massif des Maures, Var, $580 \mathrm{~m}$ d'altitude, $1200 \mathrm{~mm}$ de précipitations annuelles ; Pélenq, Haut-Var, $520 \mathrm{~m}$ d'altitude, $800 \mathrm{~mm}$ de précipitations annuelles ; La Livinière, Minervois, Hérault, $450 \mathrm{~m}$ d'altitude, $650 \mathrm{~mm}$ de précipitations annuelles. Ces échantillons couvrent la majeure partie de l'aire de répartition d' $A$. cephalonica et la partie sud de celle d'A. borisii-regis. D’autres espèces de conifères méditerranéens sont également présentes dans les stations d'essai.

Le modèle du dispositif expérimental est le même sur les 3 stations. Les plants ont été mis en place au cours de l'hiver 1970-1971 après 5 années de pépinière sur un terrain préalablement décapé et sous-solé. Le dispositif a été formé selon le schéma du lattice équilibré à blocs incomplets (Cochran and Cox, 1957). Chaque parcelle unitaire ( 4 par bloc pour 20 blocs, 5 répétitions et 16 traitements par plantation) contient un nombre variable d'individus d'une même provenance selon la plantation : 72 au Treps et à Pélenq. 60 à la Livinière. 


\section{TABleaU 1}

Quelques caractéristiques des provenances de Sapin de Grèce sensu lato présentes sur le dispositif expérimental.

Some characteristics of Greek Fir provenances in the 3 experimental sites.

\begin{tabular}{|c|c|c|c|c|}
\hline Nom & Provenance & Altitude et exposition & Substrat & Espèce \\
\hline PRIL & $\begin{array}{l}\text { Profitis Ilias, Taygète, } \\
\text { Grèce. }\end{array}$ & $\begin{array}{l}1450 \mathrm{~m} \text {, Nord. } \\
\text { Etage supramédit. }\end{array}$ & Calcaire. & A. cephalonica. \\
\hline XERO & $\begin{array}{l}\text { Xerovouna, Taygète, } \\
\text { Grèce. }\end{array}$ & $\begin{array}{l}1200 \mathrm{~m} \text {, Nord. } \\
\text { Etage supramédit. }\end{array}$ & Calcaire. & A. cephalonica. \\
\hline $\mathrm{KAPO}$ & $\begin{array}{l}\text { Kapota, Mainalon. } \\
\text { Grèce. }\end{array}$ & $\begin{array}{l}1300 \mathrm{~m} \text {, Est. } \\
\text { Etage supramédit. }\end{array}$ & $\begin{array}{l}\text { Calcaire } \\
\text { dolomitique. }\end{array}$ & A. cephalonica. \\
\hline VLAH & $\begin{array}{l}\text { Vlahica, Mainalon, } \\
\text { Grèce. }\end{array}$ & $\begin{array}{l}1300 \mathrm{~m} \text {, Nord. } \\
\text { Etage supramédit. }\end{array}$ & Calcaire. & A. cephalonica. \\
\hline LAGA & $\begin{array}{l}\text { Lagada, Mainalon, } \\
\text { Grèce. }\end{array}$ & $\begin{array}{l}750 \mathrm{~m} \text {, Nord-Ouest. } \\
\text { Etage supramédit. }\end{array}$ & Calcaire. & A. cephalonica. \\
\hline BROM & $\begin{array}{l}\text { Bromopigado, Parnasse, } \\
\text { Grèce. }\end{array}$ & $\begin{array}{l}1800 \mathrm{~m} \text {, Nord-Est. } \\
\text { Etage montagnardméd. }\end{array}$ & Calcaire. & A. cephalonica. \\
\hline KORO & $\begin{array}{l}\text { Koromilies, Parnasse, } \\
\text { Grèce. }\end{array}$ & $\begin{array}{l}1500 \mathrm{~m} \text {, Nord-Est. } \\
\text { Etage supramédit. }\end{array}$ & Calcaire. & A. cephalonica. \\
\hline KOLO & $\begin{array}{l}\text { Kolokithovrissi, } \\
\text { Parnasse, Grèce. }\end{array}$ & $\begin{array}{l}1250 \mathrm{~m} \text {, Nord. } \\
\text { Etage supramédit. }\end{array}$ & Flyschs. & A. cephalonica. \\
\hline MEVR & $\begin{array}{l}\text { Megali-Vrissi, Parnasse, } \\
\text { Grèce. }\end{array}$ & $\begin{array}{l}1220 \mathrm{~m} \text {, Sud-Ouest. } \\
\text { Etage supramédit. }\end{array}$ & Flyschs. & A. cephalonica. \\
\hline KRAS & $\begin{array}{l}\text { Kerasini, Pinde Furna, } \\
\text { Grèce. }\end{array}$ & $\begin{array}{l}1200 \mathrm{~m} \text {. Nord. } \\
\text { Etage supramédit. }\end{array}$ & Flyschs. & A. borisii-regis. \\
\hline PALE & $\begin{array}{l}\text { Paleoturnos, Pinde Fur- } \\
\text { na, Grèce. }\end{array}$ & $\begin{array}{l}900 \mathrm{~m} \text {, Nord-Ouest. } \\
\text { Etage supramédit. }\end{array}$ & Flyschs. & A. borisii-regis. \\
\hline ITAL & \multicolumn{3}{|c|}{ Italie. Provenance artificielle, probablement hybride. } & A. cephalonica. \\
\hline
\end{tabular}

Les hauteurs totales ont été collectées chaque année dans l'ensemble du dispositif sur plusieurs années de végétation. Chaque hauteur totale a donc permis de calculer la dernière pousse annuelle lui correspondant. Au Treps, où la campagne de mesure a été la plus importante, les observations ont porté sur 7 années de végétation consécutives, de 1979 à 1985. A Pélenq, elles ont porté sur trois années de végétation consécutives (de 1983 à 1985) ; l'année 1980 a été prise en compte à partir de mesures antérieures (Descroix, 1981). A la Livinière, elles ont porté sur 4 années de végétation (de 1980 à 1982 et 1985). 


\subsection{Traitement des données}

Les moyennes des hauteurs totales et des pousses annuelles sont comparées les unes aux autres au moyen d'analyses de variance et de tests de comparaisons multiples de moyennes de Duncan. Pour tenir compte de l'hétérogénéité du milieu, les moyennes sont ajustées en fonction de la fertilité du bloc avant d'être comparées.

Les comportements de chaque provenance sont ensuite caractérisés par leur "écovalence » (WRICKE, in FrEEMAN, 1973) et leur «performance relative », concept inspiré de celui de « réponse » (Eberhart and Russell, 1966).

L'écovalence permet de chiffrer la stabilité d'une provenance par rapport aux changements du milieu dans lequel elle est analysée (plantations comparatives différentes, donc sols différents; variation des conditions climatiques d'une année sur l'autre). En pratique, elle est calculée par son inverse mathématique, l'interaction ; par simplicité de langage, ces 2 termes seront ici considérés comme synonymes. Plus l'interaction est élevée et plus la provenance envisagée a un comportement instable lorsque les facteurs du milieu changent.

La réponse d'une provenance à la variation d'un facteur du milieu est théoriquement calculée à partir de coefficients de régression. Du fait de l'absence de signification statistique de la plupart d'entre eux, on a substitué à la réponse la notion de performance relative en calculant chaque année le rapport de la hauteur d'une provenance donnée sur la hauteur moyenne de l'ensemble des provenances. Une performance relative supérieure à 1 met en évidence une provenance réagissant plus fortement aux conditions du milieu (facteur climatique par exemple) que l'ensemble des autres provenances pendant l'année considérée.

Une provenance intéressante pour le reboiseur aura donc une forte croissance et une bonne stabilité interannuelle et interstationnelle.

\section{Résultats}

\subsection{Débourrement}

Afin de mettre en évidence la variabilité géographique de l'espèce par un marqueur autre que la seule croissance en hauteur, les modalités du débourrement ont été observées en 1986 au Treps sur un sous-échantillon de 3 provenances représentatives de 3 régions d'origine : KAPO pour le Mainalon, KOLO pour le Parnasse et PALE pour le Pinde. La taille de l'échantillon a été fixée à 120 individus par provenance, répartis sur deux parcelles unitaires. Chaque semaine, entre les mois d'avril et de juin, l'état du bourgeon terminal de la cime de l'arbre a été noté à l'aide d'une échelle d'appréciation en 5 niveaux inspirée de Debazac (1965 et 1967).

Les 3 provenances ont des dates de débourrement très proches (entre le 6 et le 11 mai) mais cependant différentes. La provenance KOLO est plus précoce que la provenance KAPO, elle-même plus précoce que la provenance PALE. Une analyse de variance portant sur chaque individu affecté d'une note globale caractérisant son mode plus ou moins précoce de débourrement montre que les 3 provenances diffèrent 
statistiquement (test $\mathrm{F}$ de Snédécor $=19,89$ pour 2 et 272 degrés de liberté (d.d.l.), significatif à 1 p. 100). En fait, seule la provenance KOLO est réellement plus précoce, KAPO et PALE étant assez semblables ( $F=1,5$ pour 1 et 172 d.d.l., non significatif).

Néanmoins, ces variations sont là pour rappeler que le critère de débourrement peut être un marqueur significatif. C'est de plus un critère stable. En effet, ces variations sont comparables à celles observées sur de jeunes individus en pépinière (Descroix, 1981), mais avec un écart plus faible sur les individus plus âgés (effets probables de l'âge et de la taille des individus ainsi que du milieu). Le débourrement du Sapin de Grèce tend à suivre un gradient phénologique est-précoce/ouest-tardif.

\subsection{Croissance en hauteur}

\subsection{Le Treps}

Les tests $\mathrm{F}$ de Snédécor, calculés à partir des différences de hauteurs totales et de pousses annuelles entre provenances, sont significatifs à 1 p. 100, mettant en évidence une variabilité entre les populations des différentes régions.

La comparaison des hauteurs totales et des pousses annuelles de chaque provenance (fig. 2 et 3 ) exprime clairement la dominance des provenances KOLO, ITAL et du Mainalon, à l'exception de LAGA. En effet, il existe une discordance entre les
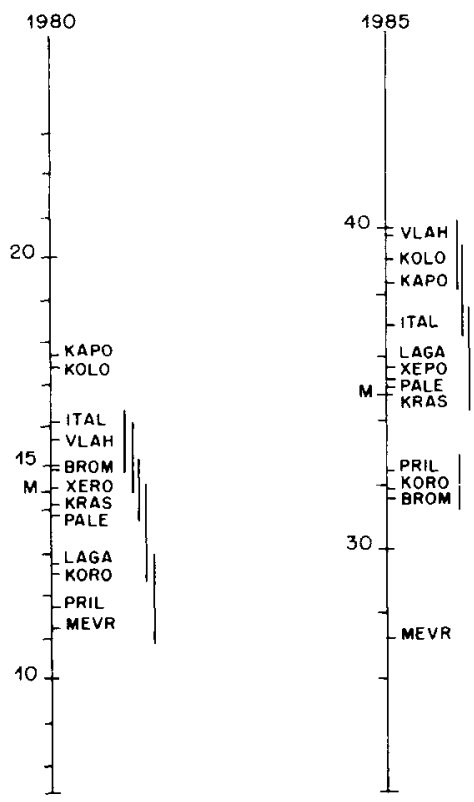

Fig. 2

Accroissements annuels (en $\mathrm{cm}$ ) et tests de Duncan (seuil de signification: 5 p. 100) des provenances d'Abies cephalonica au Treps (Maures).

$M$ correspond à la moyenne des provenances.

Annual height increments (in centimeters) and Duncan tests (signification level : 5 p. 100) of Abies cephalonica provenances in le Treps (Maures). $M$ is the provenances mean value. 

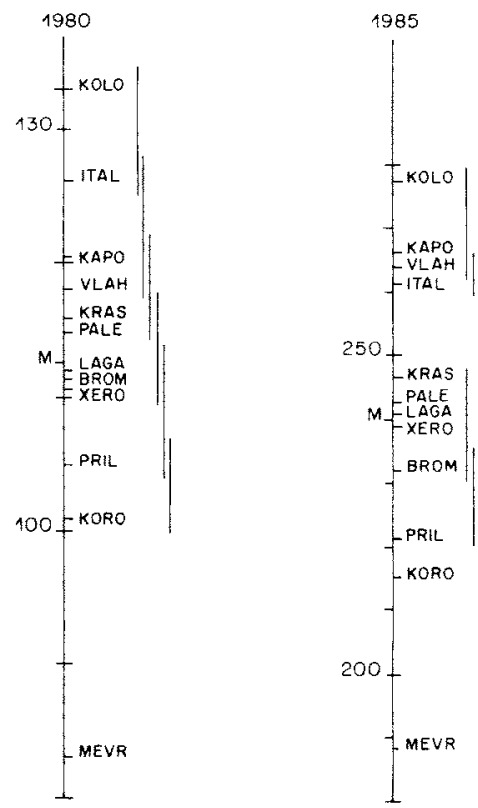

Fig. 3

Hauteurs totales (en $\mathrm{cm}$ ) et tests de Duncan (seuil de signification: $5 \mathrm{p} .100$ )

des provenances d'Abies cephalonica du Treps. $M$ correspond à la moyenne des provenances.

Total heights (in centimeters) and Duncan tests (signification level : 5 p. 100)

of Abies cephalonica provenances in le Treps. $M$ is the provenances' mean value.

résultats montrés par les deux types de figures. Si la hauteur totale donne une vision statique de la croissance en hauteur et traduit la vigueur de l'arbre à un moment précis, l'accroissement annuel au contraire caractérise la dynamique de la croissance et renseigne sur les potentialités futures de l'arbre. Ainsi, l'accélération importante de la croissance de cette provenance entre 1981 et 1985 (fig. 4) n'est pas encore apparue très nettement en 1985 sur la hauteur totale. Il convient de noter que la provenance LAGA représente un cas particulier et qu'en général les discordances dans le classement des provenances sont rares (et ce sera vrai dans l'ensemble du dispositif expérimental). La spécificité de la dynamique de croissance de chaque provenance semble donc s'acquérir relativement tôt et se conserver dans le temps.

L'écovalence interannuelle, par ses faibles valeurs (tabl. 2), indique la stabilité générale des provenances du Sapin de Grèce. L'espèce dans son ensemble est peu sensible aux variations climatiques annuelles, probablement du fait d'un contrôle endogène bien marqué de la croissance en hauteur (Ford, 1980).

L'observation des performances relatives de chaque provenance (tabl. 3) montre que seuls les individus du Mainalon (à l'exception de LAGA) ainsi que ceux de KOLO ont une valeur constamment supérieure à l'unité, caractérisant une croissance vigoureuse, supérieure à la moyenne quelles que soient les conditions climatiques de l'année. Les provenances du Pinde ainsi que celles de XERO ont un comportement proche de la moyenne (valeur de la performance relative centrée sur l'unité), ce que laissait prévoir l'observation des figures 2 et 3 . Le reste des provenances du Parnasse et du 
Ecovalence interannuelle et moyenne des pousses annuelles des provenances d'Abies cephalonica pendant 6 années au Treps.

Ecovalence and mean height increments of Abies cephalonica provenances over a 6 year period in le Treps.

\begin{tabular}{c|c|c}
\hline & Ecovalence & Hauteur $(\mathrm{cm})$ \\
\cline { 2 - 3 } PRIL & 1,32 & 21,60 \\
XERO & 1,58 & 21,80 \\
\hline LAGA & 5,04 & 22,36 \\
VLAH & 5,05 & 27,48 \\
KAPO & 2,18 & 26,84 \\
\hline MEVR & 0,58 & 21,46 \\
BROM & 0,32 & 22,58 \\
KORO & 0,96 & 23,93 \\
KOLO & 0,80 & 26,90 \\
\hline KRAS & 1,61 & 24,41 \\
PALE & 1,25 & 22,27 \\
\hline ITAL & 0,30 & 23,32 \\
\hline
\end{tabular}

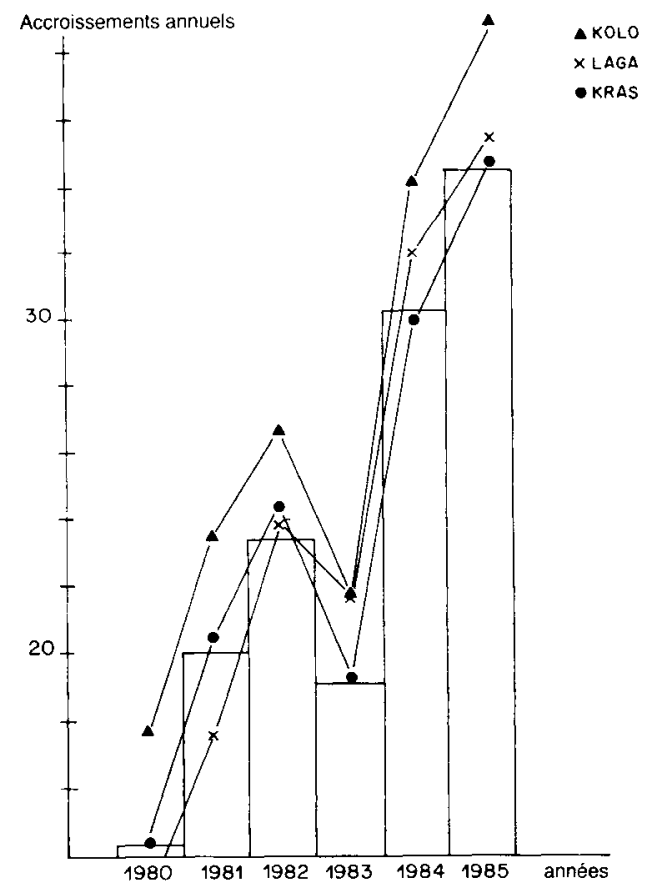

FIG. 4

Comparaison des accroissements annuels (en $\mathrm{cm}$ ) de quelques provenances caractéristiques avec l'accroissement annuel moyen de 1980 à 1985 au Treps.

Comparison of annual height increments (in centimeters) of a few characteristic provenances with mean annual height increments in Le Treps from 1980 to 1985. 
Moyenne et écart-type des performances relatives des provenances de Sapin de Grèce en fonction de la fertilité annuelle: de 1980 à 1985 au Treps; en 1980, 1984 et 1985 à Pélenq; en 1981 et 1982 à la Livinière.

Mean and standard deviation of relative performances of Greek Fir provenances : from 1980 to 1985 in le Treps; in 1980, 1984 and 1985 in Pélenq; in 1981 and 1982 in la Livinière.

\begin{tabular}{c|c|c|c|c|c|c}
\hline \multirow{2}{*}{} & \multicolumn{2}{|c|}{ Le Treps } & \multicolumn{2}{c|}{ Pélenq } & \multicolumn{2}{c}{ La Livinière } \\
\cline { 2 - 7 } & P.R. & E.T. & P.R. & E.T. & P.R. & E.T. \\
\cline { 2 - 7 } PRIL & 0,89 & 0,048 & 0,91 & 0,064 & 0,79 & 0,028 \\
XERO & 1,01 & 0,021 & 0,85 & 0,090 & 1,00 & 0,057 \\
LAGA & 1,00 & 0,096 & 1,15 & 0,100 & 1,04 & 0,007 \\
VLAH & 1,12 & 0,031 & 1,07 & 0,017 & 1,16 & 0,028 \\
KAPO & 1,15 & 0,052 & 1,14 & 0,012 & 1,05 & 0,092 \\
MEVR & 0,81 & 0,055 & 0,93 & 0,046 & 0,95 & 0,049 \\
BROM & 0,96 & 0,060 & 0,84 & 0,076 & 0,99 & 0,071 \\
KORO & 0,90 & 0,025 & 0,97 & 0,036 & 0,99 & 0,035 \\
KOLO & 1,16 & 0,040 & 0,99 & 0,029 & 0,96 & 0,035 \\
KRAS & 1,01 & 0,019 & 1,06 & 0,012 & 1,06 & 0,042 \\
PALE & 0,99 & 0,024 & 1,07 & 0,061 & 1,09 & 0,014 \\
\hline P.R. = Performance Relative Moyenne
\end{tabular}

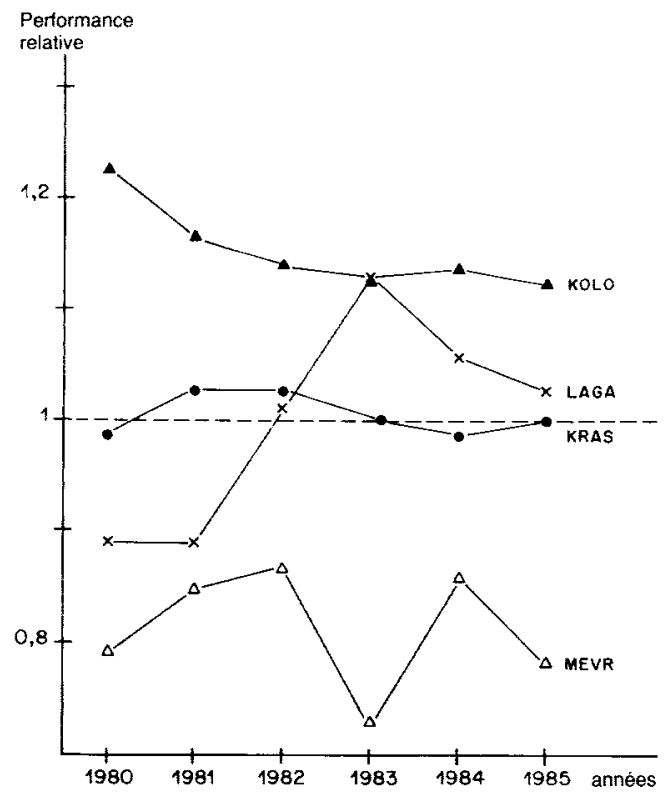

Fig. 5

Allure de la répartition des performances relatives de 4 provenances de Sapin de Grèce au Treps de 1980 à 1985.

Relative performances distribution for 4 Greek Fir provenances in Le Treps from 1980 to 1985. 
Taygète ont une vigueur systématiquement inférieure à la moyenne (valeur de la performance relative inférieure à l'unité) et sont parfois même malvenantes (MEVR par exemple).

Au cours de l'année 1983 (fig. 5), la performance relative des provenances du Mainalon se situe à leur plus haut niveau (type LAGA) ce qui n'apparaît pas chez les autres provenances qui, soit conservent une performance proche de leur moyenne (type KRAS ou KOLO), soit enregistrent une chute de leur performance relative (type MEVR). Or, cette année est caractérisée par une mauvaise croissance généralisée (fig. 4) ; elle correspond au contrecoup du déficit hydrique important de mi-avril à miseptembre 1982. Le Sapin de Grèce suivant le modèle de croissance «fixe " (CANnell, Thompson and Lines, 1976), il n'enregistre des pertes de vigueur que l'année qui suit une année de sécheresse importante.

En plus de leur hauteur moyenne élevée, les provenances du Mainalon ont donc un comportement meilleur que les autres provenances face à un stress hydrique. Les autres provenances à performance relative supérieure à l'unité (KOLO surtout mais aussi KRAS, PALE et XERO selon les années) manifestent peu ou pas d'aptitudes semblables.

\subsection{Pélenq}

Tous les tests $F$ sont significatifs à 1 p. 100 , mettant en évidence l'existence d'une variabilité génétique importante au sein de l'espèce.

Cette station est moins fertile que celle du Treps: au Treps, la hauteur totale moyenne, toutes espèces confondues, est de $240 \mathrm{~cm}$ alors qu'elle n'est que de $172 \mathrm{~cm}$ à Pélenq. De même, son dynamisme est plus faible : entre 1980 et 1985, les hauteurs totales moyennes ont doublé au Treps (de 116 à $240 \mathrm{~cm}$ ) alors qu'elles n'ont fait qu'augmenter de 70 p. 100 à Pélenq (de 101 à $172 \mathrm{~cm}$ ).

La comparaison des hauteurs totales et des pousses annuelles de chaque provenance (fig. 6 et 7 ) met en relief la vigueur importante des individus du Pinde et du Mainalon. Si les hauteurs totales montrent la croissance relativement meilleure des provenances du Pinde, les pousses annuelles les plus récentes montrent en fait une codominance des 2 régions Pinde et Mainalon. La provenance artificielle ITAL est bien située, mais la provenance KOLO est beaucoup plus moyenne que précédemment. Les provenances du Parnasse et du Taygète sont une nouvelle fois en queue de classement.

L'écovalence interannuelle n'a pas été calculée et les performances relatives (tabl. 3), calculées sur un petit nombre d'années, ne sont régulièrement supérieures à l'unité que pour les provenances du Pinde et du Mainalon, ce qui confirme les résultats acquis sur la plantation du Treps.

\subsection{La Livinière}

Les tests $\mathrm{F}$ sont significatifs à 1 p. 100 ; la variabilité spécifique est donc une nouvelle fois marquée.

Cette station est la plus fertile des trois: en 1985, la hauteur totale moyenne, toutes espèces confondues, y est de $289 \mathrm{~cm}$ contre 240 au Treps. Mais sa dynamique est comparable à celle du Treps puisqu'entre 1980 et 1985 les hauteurs totales moyennes doublent dans les deux plantations. 

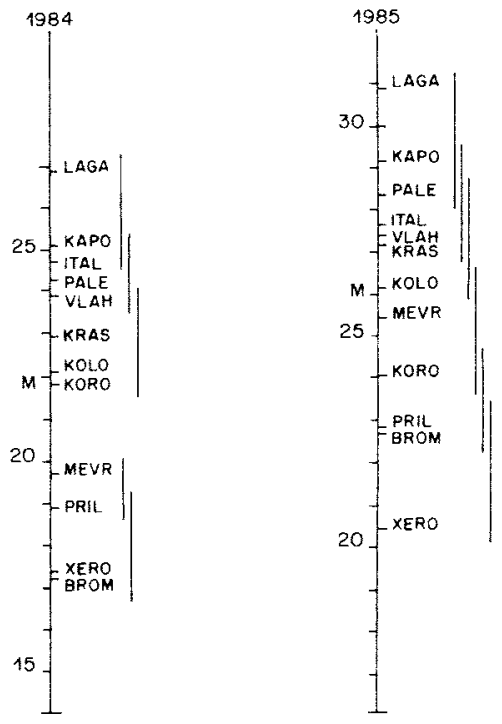

Fic. 6

Accroissements annuels (en cm) et tests de Duncan (seuil de signification : $5 p$. 100) des provenances d'Abies cephalonica à Pélenq (Haut-Var).

$M$ correspond à la moyenne des provenances.

Annual height increments (in centimeters) and Duncan tests (signification level: 5 p. 100) of Abies cephalonica provenances in Pélenq (Haut-Var). $M$ is the provenances' mean value.
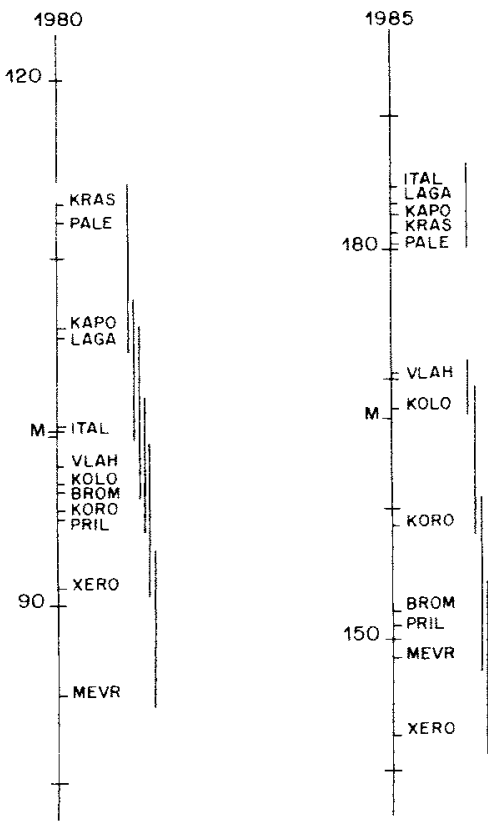

FIG. 7

Hauteurs totales (en $\mathrm{cm}$ ) et tests de Duncan (seuil de signification : 5 p. 100) des provenances d'Abies cephalonica à Pélenq. $M$ correspond à la moyenne des provenances.

Total heights (in centimeters) and Duncan tests (signification level : 5 p. 100) of Abies cephalonica provenances in Pélenq. $M$ is the provenances' mean value. 

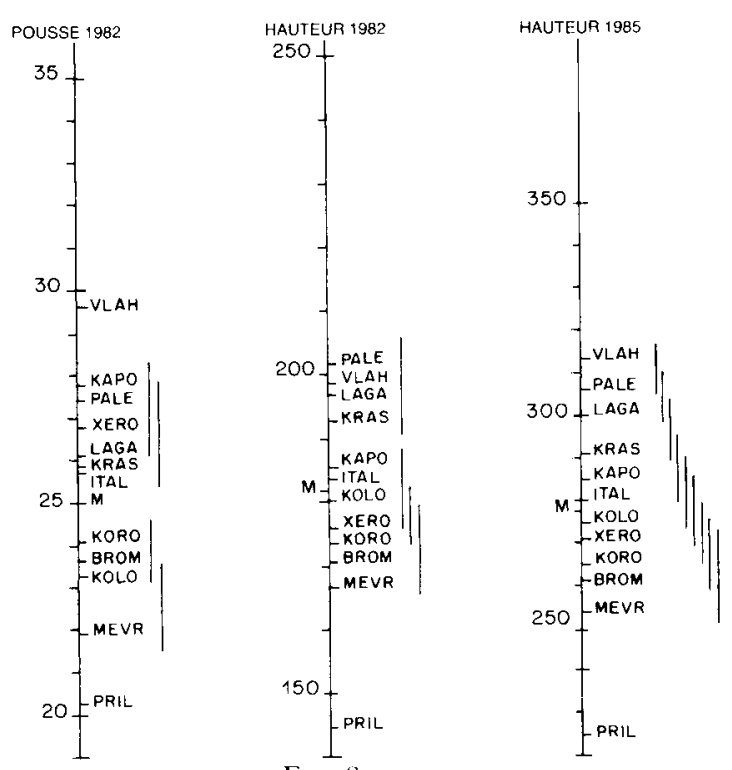

FIG. 8

Accroissements annuels (en $\mathrm{cm}$ ), hauteurs totales (en $\mathrm{cm}$ ) et tests de Duncan (seuil de signification : 5 p. 100) des provenances d'Abics cephalonica de la Livinière (Minervois). $M$ correspond à la movenne des provenances.

Annual height increments (in centimeters), total heights (in centimeters) and Duncan tests (signification level : 5 p. 100) of Abies cephalonica provenances in la Livinière (Minervois). $M$ is the provenances' mean value.

Les massifs du Pinde et du Mainalon ont les provenances les plus performantes. $\mathrm{Ni}$ les hauteurs totales ni les pousses annuelles (fig. 8) ne permettent de discriminer ces deux groupes de provenances. Comme à Pélenq, les provenances du Pinde améliorent donc leur performance par rapport au Treps. Les individus ITAL ne sont que très moyens et la provenance KOLO ne fait plus partie du groupe de tête. Les provenances du Parnasse, puis du Taygète sont les moins bien placées.

Les performances relatives supérieures à 1 (tabl. 3) sont le seul apanage des individus du Pinde et du Mainalon. Malgré le petit nombre d'années sur lequel l'expérimentation a porté, ceci confirme leur vigueur générale supérieure à la moyenne. de même que dans les plantations précédentes.

\subsection{Analyse interstationnelle}

Du fait des différences climatiques et édaphiques existant entre les 3 stations d'étude, il était intéressant de tester le comportement des diverses provenances vis-à-vis de ces variations. L'analyse comparative a été effectuée par le calcul de l'écovalence interstationnelle et de la hauteur moyenne la plus récente (1985) toutes stations confondues (fig. 9). La dispersion des provenances est significative. Les provenances ayant à la fois une grande hauteur moyenne et une grande stabilité par rapport au substrat sont celles du Mainalon et du Pinde (hauteurs totales en 1985 respectivement 


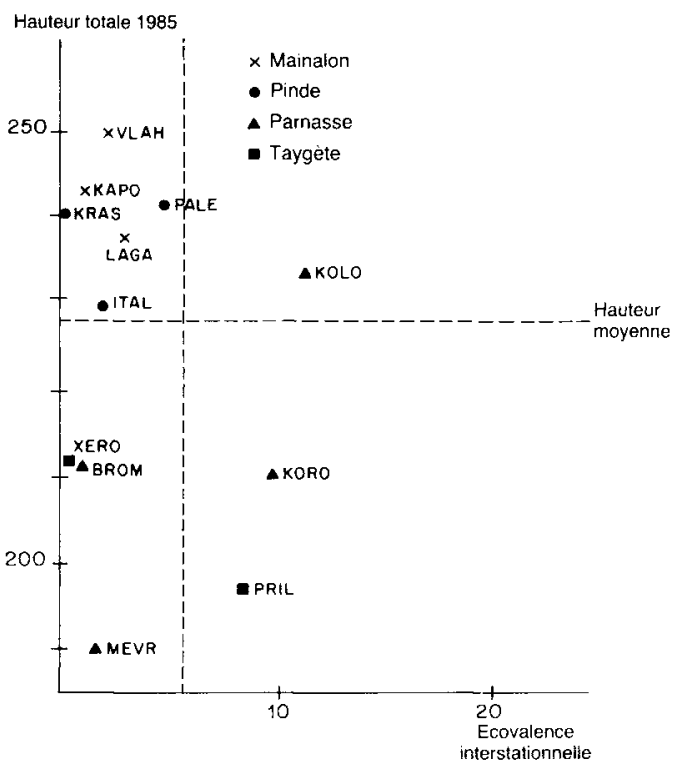

FIG. 9

Répartition des provenances d'Abies cephalonica en fonction de leur stabilité interstationnelle et de leur hauteur moyenne sur les 3 stations. Les provenances ayant une hauteur totale supérieure à la moyenne et une grande stabilité (l'écovalence n'est pas suffisamment élevée

pour avoir une signification statistique) sont contenues dans le quadrant supérieur gauche.

Distribution of Abies cephalonica provenances in relation to inter-site stability and mean total height on the 3 experimental sites. Provenances with a total height above the provenances mean height and $a$ high stability (their ecovalence has no statistical signification) appear in the top-left part.

243 et $241 \mathrm{~cm})$. La provenance ITAL, malgré sa hauteur totale supérieure à la moyenne, ne peut être prise en compte dans l'étude de la variabilité génétique à cause de son origine incertaine. Les provenances du Parnasse et du Taygète sont soit peu vigoureuses (hauteurs totales en 1985 respectivement 212 et $205 \mathrm{~cm}$ ), soit sensibles à leur situation spatiale (KOLO par exemple), soit même les deux à la fois (PRIL et même KORO par exemple).

\section{Conclusion}

La variabilité génétique du Sapin de Céphalonie sensu lato, considérant les résultats acquis sur le débourrement et la croissance en hauteur, s'avère importante et les différences entre massifs sont particulièrement bien marquées. La dynamique de croissance de chaque provenance n'offre que peu d'irrégularités au cours des années d'étude 
et les différences entre massifs se retrouvent systématiquement d'une année sur l'autre. Cette particularité peut être intéressante pour la sélection précoce, s'il s’avère qu'elle demeure au stade adulte (en 1985, les arbres ont 20 années de végétation). La croissance spatiale de l'espèce est plus variable et certaines provenances sont manifestement sensibles à un ou plusieurs facteurs du milieu. Une étude systématique des conditions bioclimatiques et édaphiques précises du lieu d'origine en comparaison avec celles du lieu de plantation permettrait sans doute de caractériser les facteurs responsables de cette variation.

Dans le cadre d'un reboisement en région méditerranéenne française, les provenances issues du Taygète et du Parnasse sont à écarter en raison, d'une part de leur manque de vigueur général et parfois de leur instabilité spatiale et d'autre part de leur grande hétérogénéité d'expression à l'intérieur d'un même massif. Néanmoins certaines d'entr'elles ont des caractéristiques intéressantes et on peut vouloir les conserver en vue d'hybridations par exemple : ainsi la provenance KOLO du Parnasse, particulièrement vigoureuse au Treps sur sol siliceux très filtrant et en bioclimat humide à sécheresse estivale accusée, mais peu performante ailleurs, ou encore la provenance XERO du Taygète, résistant bien au stress hydrique, au comportement très stable d'une station à Yautre, mais de faible vigueur.

Les provenances les plus vigoureuses, les moins sensibles aux variations climatiques et édaphiques, sont celles du Pinde et du Mainalon. Elles constituent donc un matériel de choix en vue de reboisements et d’améliorations génétiques. La réponse plus faible en année sèche des provenances du Pinde et l'écologie de leur lieu d'origine montrent qu'elles sont mieux adaptées à des conditions de milieu moins sévères (températures plus fraîches et/ou précipitations annuelles mieux réparties) que celles supportées par les provenances du Mainalon. Leur utilisation en reboisements paraît recommandée surtout dans les formations végétales de l'étage du Chêne Pubescent.

Parmi toutes les provenances échantillonnées, celles du Mainalon sont les plus rustiques, les mieux adaptées aux rigueurs du climat méditerranéen et les plus vigoureuses. Elles pourront donc fournir un apport important dans la revalorisation des forêts dégradées du sud de la France aux étages du Chêne Vert et du Chêne Pubescent.

Reçu le 25 mai 1987.

Accepté le 2 février 1988.

\section{Références bibliographiques}

Arbez M., 1967. Abies nordmanniana et Abies bornmulleriana: premiers résultats d'une étude de provenances en pépinière. Ann. Sci. For., 24 (2), 121-156.

Barbero M., Quezel P., 1976. Les groupements forestiers de Grèce centro-méridionale. Ecol. Medit., 2, 3-86.

Cannell M.G.R., Thompson S., Lines R., 1976. An analysis of inherent differences in shoot growth within some North-temperate conifers. In : "Tree physiology and yield improvement", M.G.R. Cannell and F.T. Last Eds, Academic Press, London, 173-205. 
Cochran W.G., Cox G.M., 1957. Experimental designs. John Wiley and Sons Inc., New York, $595 \mathrm{p}$.

Debazac E.F., 1965. Observations sur le débourrement et la croissance en longueur de quelques espèces de Sapin. Rev. For. Fr., 2, 120-130.

Debazac E.F., 1967. Nouvelles observations sur le débourrement et la croissance en longueur de quelques espèces de Sapin. Rev. For. Fr., 3, 183-190.

Descroix L., 1981. Variabilité génétique du Sapin de Grèce et du Sapin de Nordmann. Etude comparée de leur comportement hydrique avec le Cèdre de l'Atlas. Mémoire de fin d'études à I'E.N.I.T.E.F. Laboratoire d'amélioration des arbres forestiers, INRA, Bordeaux, 121 p. + annexes.

Eberhart S.A., Russel., W.A., 1966. Stability parameters for comparing varieties. Crop sci., 6. 36-40.

FADY B., 1986. Variabilité génétique et stabilité d'expression de la croissance du Sapin de Céphalonie. D.E.A. d'écologie "Ecosystèmes Méditerranéens Continentaux ", Université d'Aix-Marseille III, 34 p.

ForD E.D., 1980. Impact of environment on the shoot elongation of conifers : short term effects. In : "Control of shoot growth in trees", proceedings of I.U.F.R.O. meeting. New Brunswick. Canada, 107-126.

Freeman G.H., 1973. Statistical methods for the analysis of genotype-environment interactions. Heredity, 31 (3), 339-354.

Panersos C.P., 1975. Monograph of Abies cephalonica Loud. Ann. For. Zagreb, 7 (1), 1-22.

Pauty D., 1962. Aperçu sur l'écologie du Sapin de Céphalonie et de ses hybrides. Rev. For. Fr., 8-9, 755-769.

Quezel P., 1985. Les sapins du pourtour méditerranéen. For. Med., 7 (1), $27-34$.

Quezel P., Barbero M., 1985. Carte de la végétation potentielle de la région méditerranéenne ; feuille 1 : Méditerranée orientale. Editions du C.N.R.S., Paris. 